\title{
HIPERTENSÃO RENOVASCULAR
}

\author{
RENOVASCULAR HYPERTENSION
}

Celso Amodeo', Flávio Antonio de Oliveira Borelli², Oswaldo Passarelli Jr², Rui Manuel dos Santos Póvoa³

\section{RESUMO}

Estenoses da artéria renal (EAS) é um estreitamento ou bloqueio de uma artéria para os rins. Pode causar insuficiência renal e pressão alta. Fumantes e ex-fumantes têm maior risco de contrair RAS. Os homens são afetados com essa condição duas vezes mais que as mulheres. É mais comum nas idades de 50 e 70 . Colesterol alto, diabetes, excesso de peso e histórico familiar de doenças cardíacas também são fatores de risco para RAS. A pressão alta é uma causa e resultado do RAS. A causa mais comum de bloqueio da artéria renal é a arteriosclerose (espessamento e endurecimento das paredes da artéria) com acúmulo de colesterol e placa. Isso é semelhante ao que é visto nas artérias coronárias do coração, nas artérias carótidas, no cérebro e nos vasos das pernas. Apresentamos um caso de doença vascular renal em um homem diabético e ex-fumante e é apresentada uma atualização sobre a doença.

Descritores: Hipertensão; Estenoses da artéria renal; Hipertensão Renovascular; Arteriosclerose; Angioplastia; Stents.

\section{ABSTRACT}

Renal artery stenoses (RAS) is a narrowing or blockage of an artery to the kidneys. It may cause kidney failure and high blood pressure. Smokers and ex-smokers have a greater risk of getting RAS. Men are affected with this condition twice as often as women. Itss most common in the ages of 50 and 70. High cholesterol, diabetes, being overweight, and having a family history of heart disease are also risk factors for RAS. High blood pressure is both a cause and a result of RAS. The most common cause of renal artery blockages is arteriosclerosis (the thickening and hardening of artery walls) with cholesterol and plaque build-up. This is similar to what is seen in the coronary arteries of the heart, the carotid arteries to the brain and the leg vessels. We presente a case of renal vascular disease in a diabetic and ex-smoker man and an up to date about the disease is presented.

Keywords: Hypertension; Renal Artery Stenoses; Hypertension, Renovascular; Arterioscleroses; Angioplasty; Stents.

\section{IDENTIFICAÇÃO}

R.C.M., 59 anos, sexo masculino, natural de Votorantim-SP, procedente de São Paulo-SP, casado, católico, engenheiro aposentado.

\section{QUEIXA PRINCIPAL E DURAÇÃO}

Assintomático

\section{HISTÓRIA PREGRESSA DA MOLÉSTIA ATUAL}

Paciente previamente assintomático quando há 5 anos apresentou episódio de mal estar, indisposição e fraqueza. Foi diagnosticada hipertensão arterial em unidade básica de saúde e iniciado terapêutica anti-hipertensiva.

Em Julho de 2014 deu entrada em Pronto Atendimento com quadro de dispneia de início súbito associado a sudorese. Diagnosticado edema agudo de pulmão e tratado com sucesso à terapia instituída.
Foi então encaminhado a hospital de referência em cardiologia para elucidação diagnóstica e acompanhamento especializado.

Estava em uso de diurético tiazídico, bloqueador do receptor da angiotensina II e beta bloqueador.

\section{ANTECEDENTES PESSOAIS}

Hipertensão arterial, e dislipidemia. Hemorragia digestiva alta por UGD, ex-tabagista; parou de fumar há 9 anos (40 maços/ ano), apendicectomia aos 8 anos de idade. Nega álcool, etilismo e doenças cardiovasculares prévias. Em exame admissional foi informado que tinha níveis de glicose no sangue no limite superior da normalidade. Obesidade grau I (IMC - 31,22 kg/m2), CA - $99 \mathrm{~cm}$.

\section{ANTECEDENTES FAMILIARES}

Desconhece hipertensão arterial na família, doença arterial coronária, doença renal e cerebrovascular.

\footnotetext{
1. Setor de Cardiopatia Hipertensiva - Disciplina de Cardiologia - UNIFESP, São Paulo, SP, Brasil.

2. Seção de Hipertensão Arterial do Instituto Dante Pazzanese de Cardiologia, São Paulo, SP, Brasil.

3. Setor de Cardiopatia Hipertensiva - Disciplina de Cardiopatia Hipertensiva - UNIFESP, São Paulo, SP, Brasil. 


\section{EXAME FÍSICO}

- Pressão arterial: 180x120 mmHg (membro superior direito sentado)

- Pressão arterial: 179x115 mmHg (membro superior esquerdo sentado)

- Pressão arterial: 193×123 mmHg (membro superior direito posição supina)

- Pressão arterial: $180 \times 123$ mmHg (membro superior direito em posição ortostática)

- Pulso radial: 76 bpm, regular e simétrico

- Frequência respiratória: 14 ipm

- Bom estado geral e nutricional, vigil, orientado no tempo e no espaço, anictérico, acianótico, afebril, descorado +/IV.

- Pele e fâneros: sem alterações

- Mucosas: hidratadas, descoradas +/IV

- Gânglios: sem alterações

- Pescoço: estase jugular ausente, tireoide de dimensões e textura preservados, ausência de sopros cervicais.

\section{EXAME FÍSICO}

Aparelho respiratório: elasticidade e expansibilidade torácicas preservadas, som claro pulmonar a percussão, frêmito tóraco-vocal simétrico e preservado, murmúrio vesicular simétrico sem ruídos adventícios.

Aparelho cardiovascular: pulsos poplíteos e tibiais posteriores amplos, cheios e simétricos, pulso pedioso à esquerda discretamente diminuído, extremidades aquecidas e com perfusão normal. Precordio com Ictus Cordis não visível e palpável no quinto espaço intercostal esquerdo na linha axilar anterior, impulsivo $++/$ IV, abrangendo duas polpas digitais com bulhas rítmicas, normofonéticas em 2 tempos sem sopros.

Abdome: plano, timpânico com espaço de Traube livre, flácido, indolor à palpação, sem visceromegalias, ruídos hidroaéreos presentes.

Extremidades: sem edemas ou empastamentos.

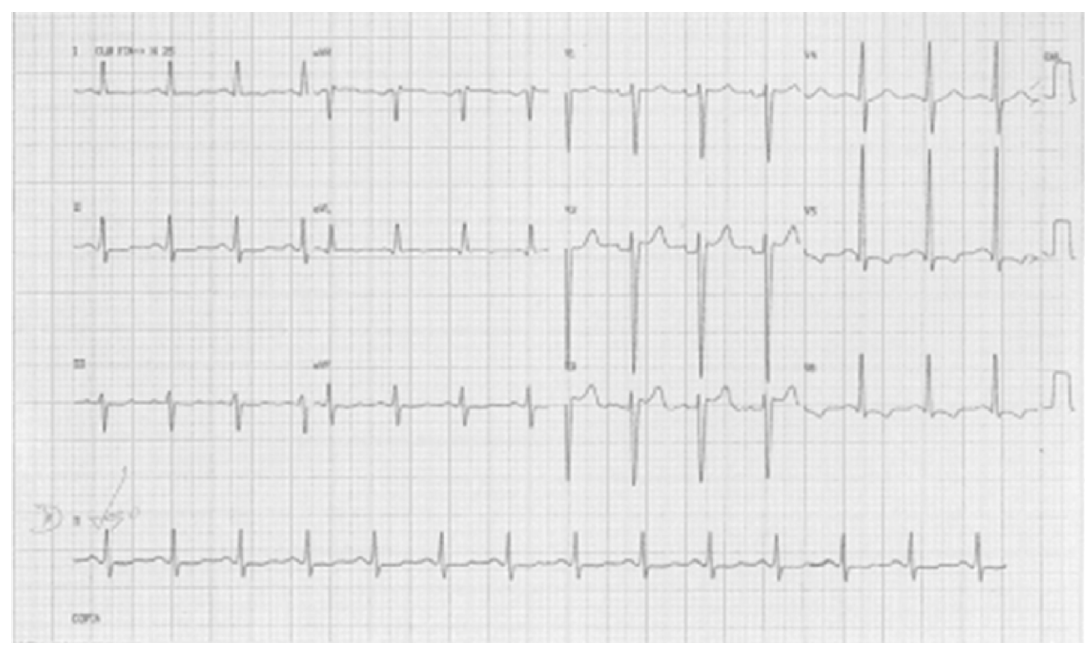

ECG: Rítmo sinusal, com eixo cardíaco desviado para esquerda e sinais de SVE pelo índice de Sokolow-Lyon.

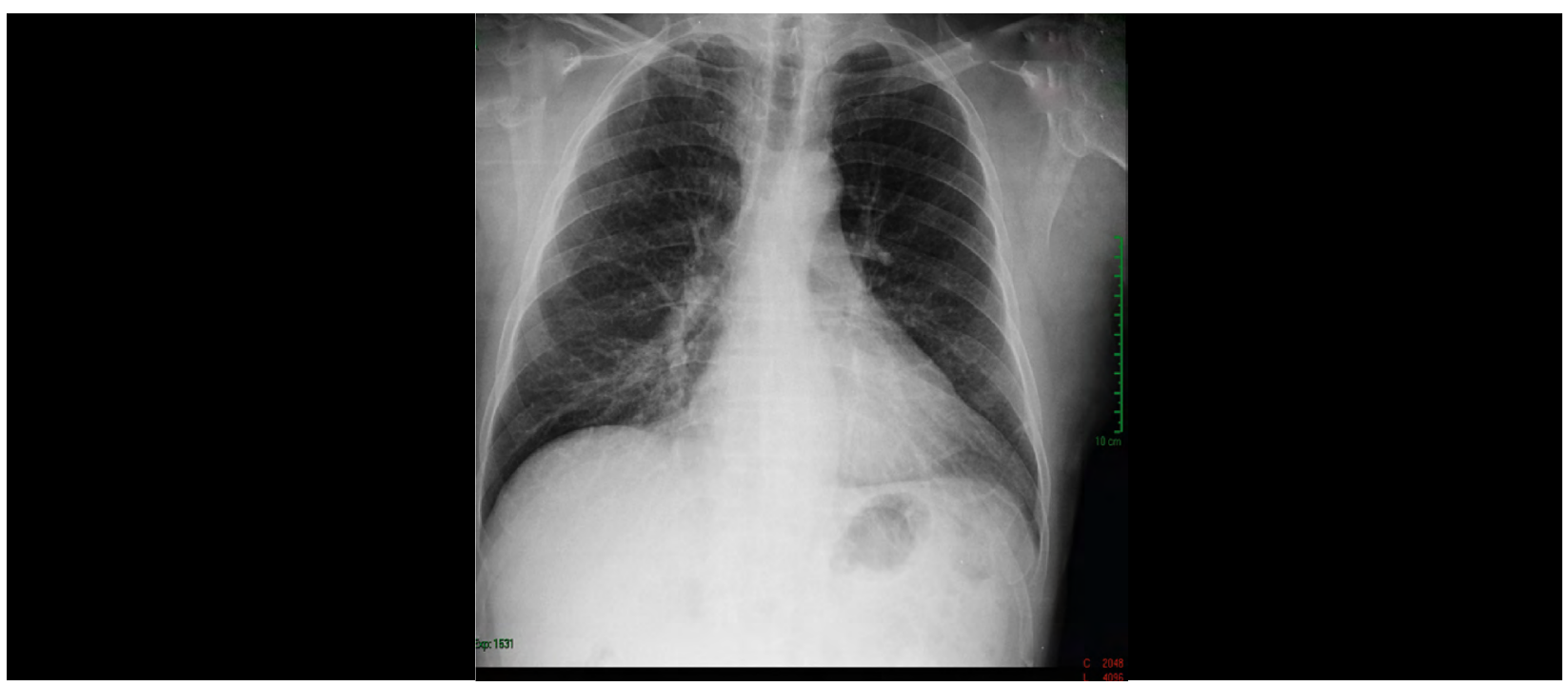




\section{CONDUTA}

Foi confirmado diabete tipo 2 (HbA1C de 8,3\%) e mantido com terapêutica específica para DM e com a associação de metformina e glicazida.

Estava em uso de três classes de anti-hipertensivos - diurético tiazídico, bloqueador do receptor AT1 da angiotensina II e um bloqueador do canal de cálcio (usava BB). Foi acrescentado espironolactona $25 \mathrm{mg} /$ dia.

\section{EVOLUÇÃO}

Retorna ao ambulatório para consulta após 60 dias.

Mantinha-se assintomático com exame clínico inalterado e pressão arterial em MSD de $160 \times 110 \mathrm{mmHg}$.

$\mathrm{Na}$ avaliação renal observou-se aumento da creatinina sérica e presença de + de proteína no exame de urina tipo 1 .

Pela história clínica, piora da função renal e hipertensão arterial resistente o paciente foi investigado para hipertensão renovascular, cujos exames demonstraram estenose $>60 \%$ na artéria renal direita. Foi submetido a angioplastia com colocação de stent em artéria renal direita. Após o procedimento que foi realizado com sucesso o paciente recebeu alta hospitalar no quarto dia após intervenção em uso das 4 classes de anti-hipertensivos (tiazídico, BRA II, ACC, espironolactona) além de sinvastatina, metformina, glicazida, AAS e clopidogrel).

$\mathrm{Na}$ alta hospitalar mantinha níveis de pressão arterial de $140 \times 90 \mathrm{mmHg} \mathrm{K}-3,9 \mathrm{mEq} / \mathrm{dl}, \mathrm{C}-1,5 \mathrm{mg} / \mathrm{dl}$.

Após 20 dias retorna ao ambulatório com pressão arterial de 142 x $90 \mathrm{mmHg}$ recebendo mesma carga de anti-hipertensivos.

\begin{tabular}{|c|c|c|c|c|c|c|c|c|c|c|c|c|c|c|}
\hline & $\begin{array}{c}\text { Glicose } \\
\mathrm{mg} / \mathrm{dl}\end{array}$ & $\begin{array}{c}\text { Hbglic } \\
\%\end{array}$ & $\begin{array}{c}\text { Creatinina } \\
\mathrm{mg} / \mathrm{dl}\end{array}$ & $\begin{array}{l}\text { Hemoglobina } \\
\mathrm{g} / \mathrm{dl}\end{array}$ & Urina I & $\begin{array}{c}\mathrm{K} \\
\mathrm{meq} / \mathrm{dl}\end{array}$ & $\begin{array}{c}\text { Ac } \\
\text { úrico }\end{array}$ & $\begin{array}{c}\mathrm{Tg} \\
\mathrm{mg} / \mathrm{dl}\end{array}$ & $\begin{array}{c}\mathrm{Hdl} \\
\mathrm{mg} / \mathrm{dl}\end{array} \mid$ & $\begin{array}{l}\text { Ldl } \\
\text { mg/dl }\end{array}$ & $\begin{array}{c}\text { Meta } \\
\text { nefrina } \\
\text { Urinária } \\
\text { mcg/24h }\end{array}$ & $\begin{array}{c}\text { Catecol } \\
\text { Plasm } \\
\text { pg/ml }\end{array}$ & $\begin{array}{c}\text { Aldostero } \\
\text { sérica } \\
\text { ng/dl }\end{array}$ & $\begin{array}{l}\text { Renina } \\
\text { sérica } \\
\text { pg/ml }\end{array}$ \\
\hline 2014 & 108 & 8,3 & 1,4 & 13,9 & NL & 4,4 & 5,8 & 268 & 36 & 128 & & & & \\
\hline 2014 & 86 & 7,0 & 1,4 & 12,5 & NL & 4,1 & 6,7 & 156 & 40 & 96 & & & & \\
\hline 2014 & 90 & 6,5 & 2,4 & 11,5 & Proteinúria + & 4,0 & 6,5 & 150 & 41 & 90 & & & & \\
\hline 2014 & 90 & 6,5 & 2,6 & 11,8 & $\begin{array}{c}\text { Proteinúria } \\
+\end{array}$ & 4,2 & 6,4 & 148 & 44 & 89 & 56 & $\begin{array}{l}\text { A-28.49/ } \\
\text { N-97.21/ } \\
\text { D-52.57 }\end{array}$ & 18 & 21 \\
\hline
\end{tabular}
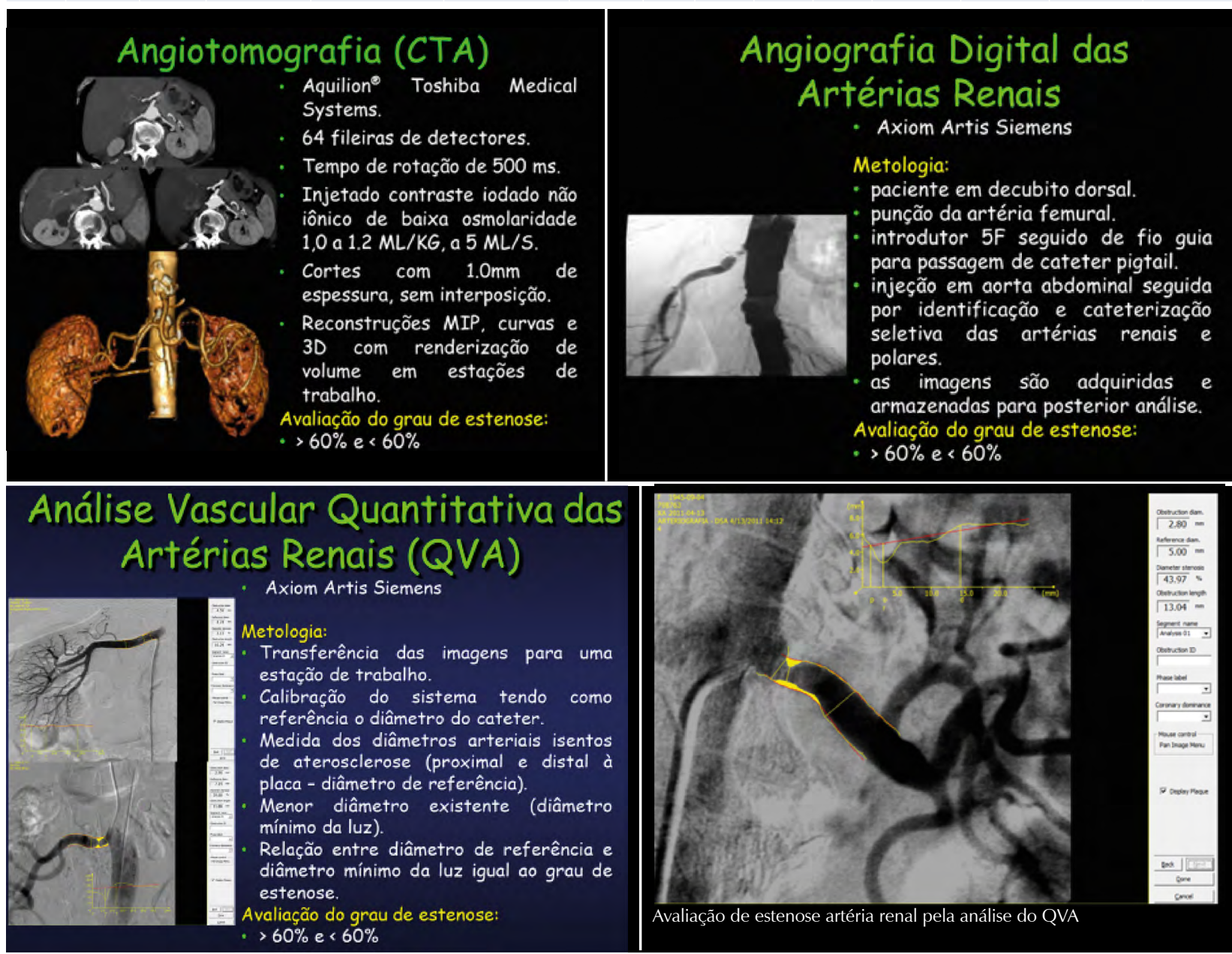

Arquivos: Instituto Dante Pazzanese de Cardiologia 


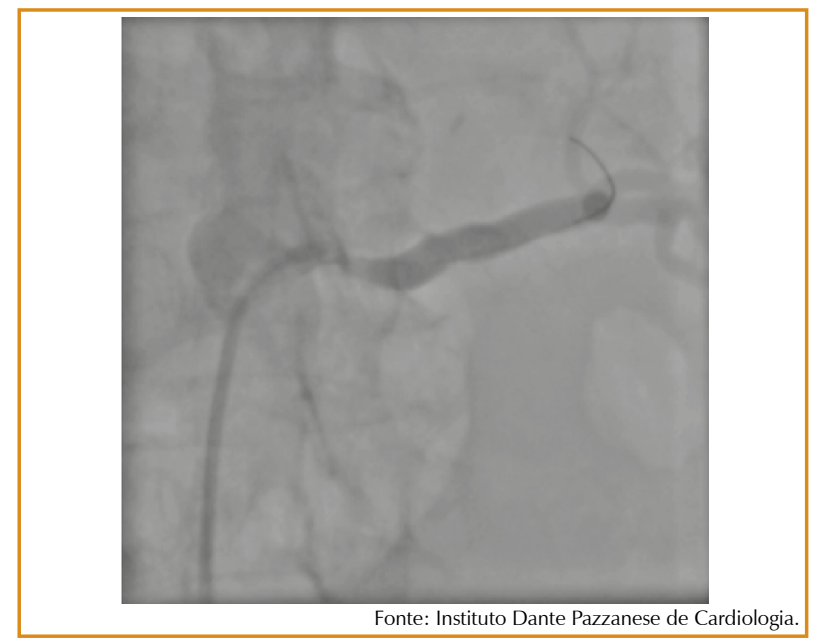

Figura 1. Lesão suboclusiva no terço próxima da artéria renal esquerda.

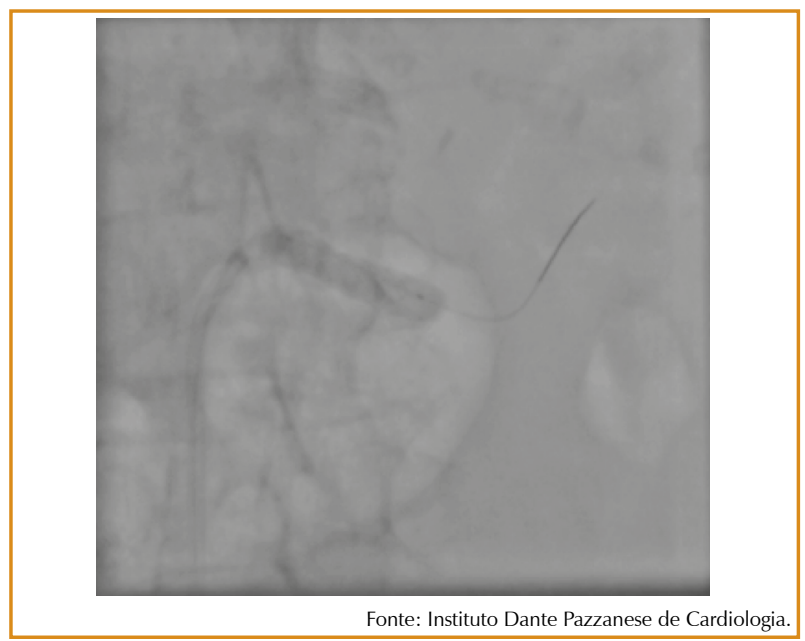

Figura 2. Balão insuflado para implante do Stent cobrindo a lesão existente.

\section{DISCUSSÃO}

A doença renovascular é uma das causas mais comuns de hipertensão secundária mas, é responsável por $<2 \%$ de todos os casos de hipertensão. Estenose ou oclusão de uma artéria renal principal, artéria renal acessória ou qualquer um dos seus ramos pode causar hipertensão pela estimulação da liberação de renina das células justaglomerulares do rim comprometido. Em geral, a magnitude da estenose arterial renal deve ser $\geq$ $70 \%$ e um gradiente pós-estenótico significativo deve estar presente antes que provavelmente a estenose contribua para uma elevação da PA.

De maneira geral, cerca de $80 \%$ dos casos são provocados por aterosclerose e $20 \%$ por displasia fibromuscular. A aterosclerose é mais comum em homens com $>50$ anos de idade, comprometendo principalmente o terço proximal da artéria renal. A displasia fibromuscular é mais comum em pacientes mais jovens (em geral, mulheres) e, normalmente, compromete os dois terços distais da artéria renal principal e os ramos das artérias renais.

Este caso clínico apresentado ilustra uma situação de

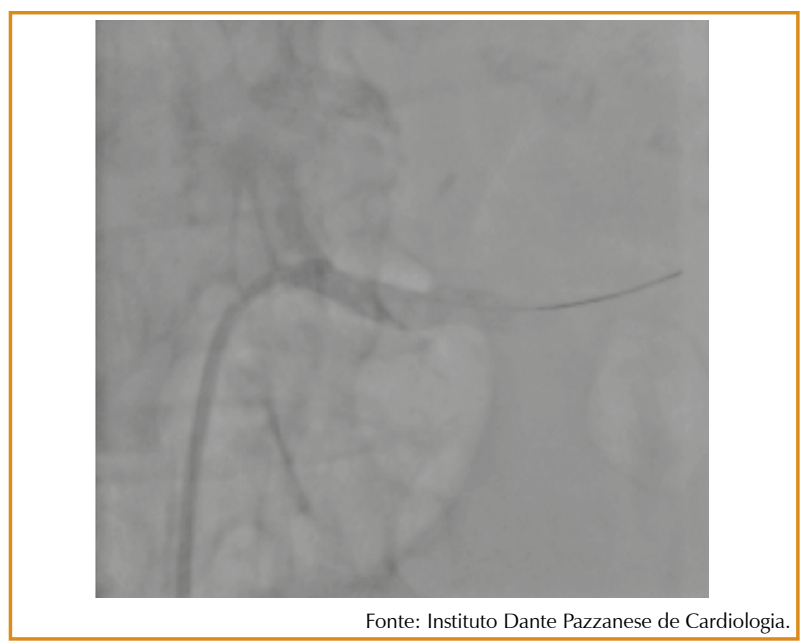

Figura 3. Restituição da luz do vaso.

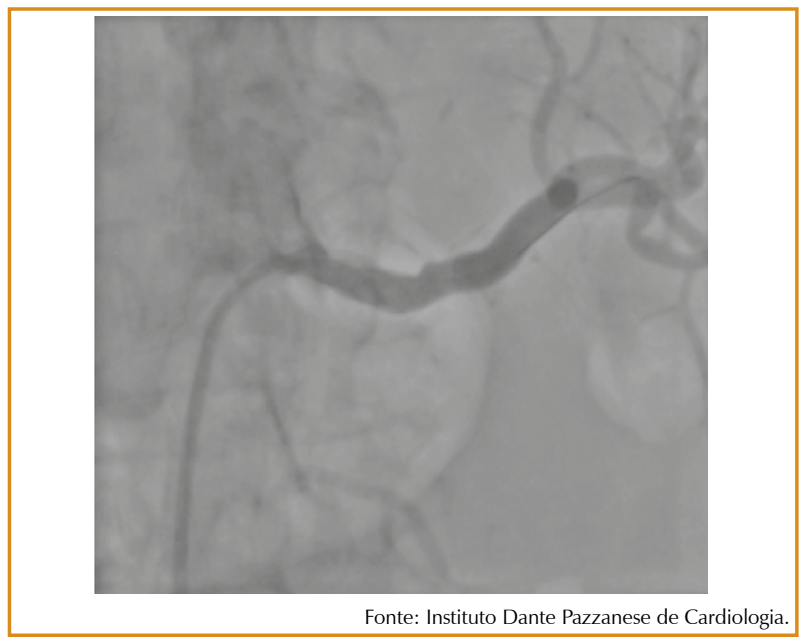

Figura 4. Observa-se a totalidade do vaso bem preenchida com o meio de contraste e as porções finais bem definidas.

estenose de artéria renal contribuindo para o não controle da pressão arterial e produção de quadro clínico de edema agudo de pulmão. Paciente de 59 anos com história de hipertensão arterial há mais de 5 anos que no início controlava com duas classes de medicamentos anti-hipertensivos e que, três anos após o diagnósticos necessitava de mais classes de fármacos sem controle adequado da pressão arterial. Foi então classificado como hipertenso resistente, visto que o mesmo estava sob uso de quatro classes de fármacos em doses adequadas sem controle da pressão arterial. A presença de alteração na creatinina sérica, diabetes e edema agudo de pulmão levantaram a hipótese de hipertensão arterial renovascular.

A Tabela 1 abaixo apresenta as situações onde se deve suspeitar de hipertensão renovascular.

Mesmo após a correção do estreitamento da artéria renal o paciente persistiu com hipertensão arterial necessitando também 4 classes de fármacos. Entretanto os níveis pressóricos, embora ainda elevados estavam mais baixos que antes da dilatação da artéria renal. Isso chama a atenção de tratar-se de um hipertenso primário já com lesão de órgão 
Tabela 2. indicadores clínicos de probabilidade de hipertensão renovascular e proposta de investigação.

\begin{tabular}{|c|c|}
\hline Probabilidade & Características clínicas \\
\hline \multirow{5}{*}{$\begin{array}{l}\text { Baixa } \\
(0,2 \%)\end{array}$} & Hipertensão limítrofe \\
\hline & Hipertensão leve/moderada não complicada \\
\hline & Hipertensão grave ou refratária \\
\hline & $\begin{array}{l}\text { Hipertensão recente abaixo dos } 30 \text { ou acima } \\
\text { dos } 50 \text { anos }\end{array}$ \\
\hline & Presença de sopros abdominais ou lombares \\
\hline \multirow{5}{*}{$\begin{array}{l}\text { Média } \\
(5-15 \%)\end{array}$} & Assimetria de pulsos radiais ou carotídeos \\
\hline & $\begin{array}{l}\text { Hipertensos moderados tabagistas ou com } \\
\text { aterosclerose em outro local (coronariana ou } \\
\text { carotidea) }\end{array}$ \\
\hline & Déficit de função renal não definido \\
\hline & Resposta pressórica exagerada aos iECA \\
\hline & $\begin{array}{l}\text { Hipertensão grave ou refratária com } \\
\text { insuficiência renal progressiva }\end{array}$ \\
\hline \multirow{3}{*}{$\begin{array}{c}\text { Alta } \\
(25 \%)\end{array}$} & Hipertensão acelerada ou maligna \\
\hline & Aumento da creatinina induzida por iECA \\
\hline & Assimetria de tamanho ou função renal \\
\hline
\end{tabular}

alvo (disfunção renal e hipertrofia ventricular esquerda) que apresentava um componente secundário sobre a sua hipertensão arterial primária não se caracterizam como hipertensão renovascular.

Vale ressaltar que a maioria dos casos de hipertensão arterial com estenose de artéria renal não apresentam hipertensão arterial renovascular. Quando identificamos uma estenose de artéria renal devemos analisar os valores de pressão arterial e a quantidade de medicamentos que o paciente esta tomando. Também se deve monitorizar a função renal e o tamanho ou volume renal direito e esquerdo. Se a pressão arterial fica controlada com as medicações em uso, a função renal esta estável e o volume renal se mantém a decisão terapêutica deve ser o seguimento clínico com avaliações periódicas desses parâmetros clínico-laboratoriais. Entretanto, se a pressão arterial se torna de difícil controle, ou a função renal piora ou o volume renal diminui, deve-se suspeitar de um componente renovascular na etiologia da hipertensão do paciente.

No caso deste paciente a dificuldade em se controlar a pressão arterial (hipertensão resistente) e a presença de edema agudo de pulmão sem causa aparente nos levaram à conduta de encaminhamento para estudo das artérias renais. Uma vez diagnosticado o estreitamento importante da arterial renal na vigência dessa clínica apresentada o paciente foi submetido à dilatação da arterial renal.

Mesmo após a dilatação o paciente evoluiu com valores de pressão arterial elevadas em menor nível, caracterizando a hipertensão arterial primária como uma possibilidade e o mesmo foi mantido em esquema terapêutico das diversas comorbidades apresentadas como descrito na apresentação deste caso clínico.

\section{LEITURA RECOMENDADA}

1. Goldblatt H, Lynch J, Hanzal RF, Summerville WW. Studies on Experimental Hypertension: I. The Production of Persistent Elevation of Systolic Blood Pressure by Means of Renal Ischemia. J Exp Med. 1934; 59(3):347-79.

2. Pickering TG. Diagnosis and evaluation of renovascular hypertension. Indications for therapy. Circulation. 1991;83(2 Suppl):1147-54.

3. Textor SC. Revascularization in atherosclerotic renal artery disease. Kidney Int. 1998; 53(3):799-811.

4. Lim ST, Rosenfield K. Renal Artery Stent Placement: Indications and Results. Curr Interv Cardiol Rep. 2000;2(2):130-9.

5. Gross CM, Kramer J, Weingartner O, et al. Determination of renal arterial stenosis severity: comparison of pressure gradient and vessel diameter. Radiology. 2001;220(3):751- 6 .

6. Zierler RE. Screening for renal artery stenosis: is it justified? Mayo Clin Proc. 2002; 77:307-8

7. Nordmann AJ, Woo K, Parkes R, Logan AG. Ballon angioplasty or medical therapy for hypertensive patients with atherosclerotic renal artery stenosis? A meta-analysis of randomized controlled trials. Am J Med. 2003;114:44-50.

8. Wheatley K, Ives N, Gray R, Kalra PA, Moss JG, Baigent C, et al; ASTRAL Investigators. Revascularization vs. medical therapy for renal-artery stenosis. N Engl J Med. 2009;361(20):1953-62.

9. Borelli FAO, Pinto IMF, Amodeo C, et al. Avaliação da Sensibilidade e

Especificidade dos Exames Não Invasivos no Diagnóstico da Estenose de Artéria Renal. Arq Bras Cardiol. 2013;101(5):423-33.

10. Cooper CJ, Murphy TP, Cutlip DE, et al. Stenting and medical therapy for atherosclerotic renal-artery stenosis. N Engl J Med. 2014;370:13-22.

11. Cooper CJ, Murphy TP, Cutlip DE, et al; CORAL Investigators. Stenting and medical therapy for atherosclerotic renal-artery stenosis. N Engl J Med. 2014;370(1):13-22.

12. 7aㅡ Diretriz Brasileira de Hipertensão Arterial. Arq Bras Cardiol. 2016; 107(3Supl.3):1-83.

13. de Leeuw PW, Postma CT, Spiering W, Kroon AA. Atherosclerotic Renal Artery Stenosis: Should we Intervene Earlier? Curr Hypertens Rep. 2018;20(4):35. Matayoshi T, Kamide K, Tanaka R, et al. Factors Associated with Outcomes of Percutaneous Transluminal Renal Angioplasty in Patients with Renal Artery Stenosis: A Retrospective Analysis of 50 Consecutive Cases. Int J Hypertens. 2018 Jan 4;2018:1952685. doi: 10.1155/2018/1952685.

14. Sardar P, Giri J, Jaff MR, et al. Strength of Evidence Underlying the American Heart Association/American College of Cardiology Guidelines on Endovascular and Surgical Treatment of Peripheral Vascular Disease. Circ Cardiovasc Interv. 2019 Jan;12(1):e007244. .

15. Courand et al. Renal Artery Stenosis in Resistant Hypertension. https://www. ahajournals.org/doi/suppl/10.1161/HYPERTENSIONAHA.119.13393. 